\title{
On the Orlicz Minkowski Problem for Polytopes
}

\author{
Qingzhong Huang • Binwu He
}

Received: 21 August 2010 / Revised: 3 May 2011 / Accepted: 11 June 2012 /

Published online: 23 June 2012

(C) Springer Science+Business Media, LLC 2012

\begin{abstract}
Quite recently, an Orlicz Minkowski problem has been posed and the existence part of this problem for even measures has been presented. In this paper, the existence part of the Orlicz Minkowski problem for polytopes is demonstrated. Furthermore, we obtain a solution of the Orlicz Minkowski problem for general (not necessarily even) measures.
\end{abstract}

Keywords Convex polytope $\cdot$ Orlicz norm $\cdot$ Minkowski problem

\section{Introduction}

The solution of the classical Minkowski problem for convex bodies is a central topic in convexity with many applications. Although the solution of the Minkowski problem has been known in the mathematical literature since the work of Minkowski [44, 45], Alexandrov [1-3], Fenchel and Jessen [10], analytic versions or algorithmic issues of the problem are still subject of current research and highly relevant (see, e.g., Chou and Wang [7], Jerison [21], Klain [23], Lamberg [24], Lamberg and Kaasalainen [25], and the reference therein).

The $L_{p}$ Minkowski problem extends the classical Minkowski problem, which was first defined by Lutwak in [30] as part of the $L_{p}$ Brunn-Minkowski theory (see, e.g., $[4,5,8,13-16,20,26-40,43,46-48,50,55,56])$. It requires necessary and sufficient conditions on a Borel measure $\mu$ on $S^{n-1}$ to be the $L_{p}$ surface area measure of a convex body; i.e., is there a convex body $K$ such that

$$
h_{K}^{1-p} d S_{K}=d \mu ?
$$

\footnotetext{
Q. Huang · B. He (凶)

Department of Mathematics, Shanghai University, Shanghai, 200444, P.R. China

e-mail: hebinwu@shu.edu.cn

Q. Huang

e-mail: hqz376560571@ @su.edu.cn
} 
Here, $h_{K}$ is the support function of $K$ and $S_{K}$ is the surface area measure of $K$. The even $L_{p}$ Minkowski problem asks which even measures are $L_{p}$ surface area measures of convex bodies. For $p=1$, this problem reduces to the classical Minkowski problem. For $p>1$ but $p \neq n$, the even $L_{p}$ Minkowski problem was solved by Lutwak [30]. However, in [36] an equivalent volumenormalized version of the $L_{p}$ Minkowski problem was proposed, and the even volume-normalized $L_{p}$ Minkowski problem was solved for all $p>1$. The solution of the $L_{p}$ Minkowski problem for polytopes for all $p>1$ was given by Chou and Wang [8], while an alternate approach to this problem was presented by Hug et al. [20]. Other approaches towards the $L_{p}$ Minkowski problem have also been extensively studied over the last years (see, e.g., [6, 9, 17, 19, 22, 51-54]). Despite impressive success in this direction, not all problems concerning the $L_{p}$ Minkowski problem are completely solved.

Quite recently, an embryonic Orlicz-Brunn-Minkowski theory emerged in a series of papers [18, 41, 42]. The Orlicz Minkowski problem is a natural and important task to be considered. Haberl, Lutwak, Yang and Zhang [18] first proposed the following Orlicz Minkowski problem: what are necessary and sufficient conditions for a Borel measure $\mu$ on $S^{n-1}$ to be the Orlicz surface area of a convex body; i.e., given a suitable continuous function $\varphi:(0,+\infty) \rightarrow(0,+\infty)$, is there a convex body $K$ such that for some $c$

$$
c \varphi\left(h_{K}\right) d S_{K}=d \mu ?
$$

In the case that $\varphi(t)=t^{1-p}(p \neq n)$, this problem reduces to $L_{p}$ Minkowski problem.

Under some suitable conditions on $\varphi$, the even Orlicz Minkowski problem was solved by Haberl, Lutwak, Yang and Zhang in [18]. One of their results [18, Theorem 2] is the following: Suppose $\varphi:(0, \infty) \rightarrow(0, \infty)$ is a continuous function such that $\phi(t)=\int_{o}^{t} \frac{1}{\varphi(s)} d s$ exists for every positive $t$ and is unbounded as $t \rightarrow \infty$, and $\mu$ is an even finite Borel measure on $S^{n-1}$ which is not concentrated on a great subsphere of $S^{n-1}$, then there exists an origin symmetric convex body $K \subset \mathbb{R}^{n}$ and $c>0$ such that $c \varphi\left(h_{K}\right) d S_{K}=d \mu$ and $\left\|h_{K}\right\|_{\phi, \mu}=1$ simultaneously, where $\left\|h_{K}\right\|_{\phi, \mu}$ is the Orlicz norm of the support function $h_{K}$ with respect to $\mu$.

The main purpose of this paper is to provide a solution of the general Orlicz Minkowski problem without assuming that $\mu$ is an even measure. But besides the assumptions on $\varphi$ in [18], we have to assume that $\varphi(s)$ tends to infinity as $s \rightarrow 0^{+}$. In order to serve this purpose, we first solve the Orlicz Minkowski problem for discrete measures, that is, if $\mu$ is a discrete measure then the solution body $K$ is a polytope (Theorem 1.1). The proof relies on techniques developed by Haberl, Hug, Lutwak, Yang, and Zhang in [18] and [20], but it is not just an immediate generalization. Next, we give a solution of the Orlicz Minkowski problem for general measures (Theorem 1.2), by means of an approximation argument.

We denote by $\mathcal{K}^{n}$ the set of convex bodies in $\mathbb{R}^{n}, \mathcal{P}_{0}^{n}$ the set of convex polytopes in $\mathbb{R}^{n}$ which contain the origin in their interiors, $h(P, \cdot)$ the support function of $P$, $S(P, \cdot)$ the surface area measure of $P, \delta_{u_{i}}$ the probability measure with unit point mass at $u_{i}$. One can consult Sects. 2 and 3 for more details.

Our main result may be formulated as follows: 
Theorem 1.1 (Discrete measures) Suppose $\varphi:(0, \infty) \rightarrow(0, \infty)$ is a continuous function such that $\phi(t)=\int_{o}^{t} \frac{1}{\varphi(s)} d$ s exists for every positive $t$ and is unbounded as $t \rightarrow \infty$, and $\varphi(s)$ tends to infinity as $s \rightarrow 0^{+}$. Let vectors $u_{1}, \ldots, u_{m} \in S^{n-1}$ that are not contained in a closed hemisphere and real numbers $\alpha_{1}, \ldots, \alpha_{m}>0$ be given. Then, there exist a polytope $P \in \mathcal{P}_{0}^{n}$ and $c>0$ such that

$$
\begin{aligned}
\sum_{i=1}^{m} \alpha_{i} \delta_{u_{i}} & =c \varphi(h(P, \cdot)) S(P, \cdot), \\
\left\|h_{P}\right\|_{\phi, \mu} & =1 .
\end{aligned}
$$

Theorem 1.2 (General measures) Suppose $\varphi:(0, \infty) \rightarrow(0, \infty)$ is a continuous function such that $\phi(t)=\int_{o}^{t} \frac{1}{\varphi(s)} d s$ exists for every positive $t$ and is unbounded as $t \rightarrow \infty$, and $\varphi(s)$ tends to infinity as $s \rightarrow 0^{+}$. Let $\mu$ be a finite Borel measure on $S^{n-1}$ whose support is not contained in a closed hemisphere. Then there exist a convex body $K \in \mathcal{K}^{n}$ with $0 \in K$ and $c>0$ such that

$$
\begin{aligned}
\frac{d \mu}{\varphi(h(K, \cdot))} & =c d S(K, \cdot), \\
\left\|h_{K}\right\|_{\phi, \mu} & =1 .
\end{aligned}
$$

However, just as the case of even measures, the uniqueness part of the Orlicz Minkowski problem for general measures cannot also be solved. Hence, this problem is still open and seems to be very difficult.

This paper is organized as follows: In Sect. 2 we list for quick reference some basic facts regarding convex bodies. In Sect. 3 we study some properties of the Orlicz norm on the basis of [18]. The proofs of Theorem 1.1 and Theorem 1.2 are presented in Sects. 4 and 5, respectively.

\section{Background and Notation}

In this section we present the terminology and notation we shall use throughout. For general reference the reader may wish to consult the books of Gardner [11], Gruber [12], and Schneider [49].

For $x, y \in \mathbb{R}^{n}$, we denote their inner product by $x \cdot y$ and the Euclidean norm of $x$ by $|x|=\sqrt{x \cdot x}$. The unit sphere $\left\{x \in \mathbb{R}^{n}:|x|=1\right\}$ is denoted by $S^{n-1}$ and the unit ball $\left\{x \in \mathbb{R}^{n}:|x| \leq 1\right\}$ by $B_{2}^{n}$. For $u \in S^{n-1}$, let $H_{u, t}^{-}:=\left\{y \in \mathbb{R}^{n}: u \cdot y \leq t\right\}$ denote the halfspace with exterior normal vector $u$ and the distance $t \geq 0$ from the origin.

Let $V$ stand for $n$-dimensional Lebesgue measure, and $|\mu|:=\mu\left(S^{n-1}\right)$ for a finite Borel measure $\mu$ on $S^{n-1}$. We write $C\left(S^{n-1}\right)$ for the set of continuous functions on $S^{n-1}$ which will always be viewed as equipped with the max-norm metric:

$$
\|f-g\|_{\infty}=\max _{u \in S^{n-1}}|f(u)-g(u)|,
$$

for $f, g \in C\left(S^{n-1}\right)$. 
A convex body is a compact convex set of $\mathbb{R}^{n}$ with nonempty interior. Let $\mathcal{K}^{n}$ denote the set of convex bodies in $\mathbb{R}^{n}$ endowed with the Hausdorff metric, and $\mathcal{P}^{n}$ the subset of convex polytopes. We write $\mathcal{K}_{0}^{n}$ for the set of convex bodies containing the origin in their interiors, and $\mathcal{P}_{0}^{n}:=\mathcal{P}^{n} \cap \mathcal{K}_{0}^{n}$.

For $K \in \mathcal{K}^{n}$, let $h_{K}=h(K, \cdot): \mathbb{R}^{n} \rightarrow \mathbb{R}$ denote the support function of $K$; i.e., for any $x \in \mathbb{R}^{n}, h_{K}(x)=h(K, x)=\max \{x \cdot y: y \in K\}$. It is easy to show that the support function of the line segment $\hat{v}$ joining the points $0, v \in \mathbb{R}^{n}$ is given by

$$
h_{\hat{v}}(u)=(u \cdot v)_{+}=\max \{u \cdot v, 0\}=\frac{|u \cdot v|+u \cdot v}{2}, \quad u \in \mathbb{R}^{n} .
$$

We shall require the obvious facts that for compact, convex $K, L \subset \mathbb{R}^{n}$,

$$
K \subset L \quad \text { if and only if } h_{K} \leq h_{L} \text {, }
$$

and that for $c>0$ and $x \in \mathbb{R}^{n}$,

$$
h_{c K}(x)=c h_{K}(x) \quad \text { and } \quad h_{K}(c x)=c h_{K}(x) .
$$

If $K_{i} \in \mathcal{K}^{n}$, we say that $K_{i} \rightarrow K \in \mathcal{K}^{n}$ in the Hausdorff metric provided

$$
\left\|h_{K_{i}}-h_{K}\right\|_{\infty}:=\max _{u \in S^{n-1}}\left|h_{K_{i}}(u)-h_{K}(u)\right| \rightarrow 0 .
$$

For a Borel set $\omega \subset S^{n-1}$, the surface area measure $S_{K}(\omega)=S(K, \omega)$ of the convex body $K$ is the $(n-1)$-dimensional Hausdorff measure of the set of all boundary points of $K$ for which there exists a normal vector of $K$ belonging to $\omega$. Observe that for the surface area measure of $c K$ we have

$$
S_{c K}=c^{n-1} S_{K}, \quad c>0 .
$$

We will use the fact that $S_{K}$ is weakly continuous in $K$ [49]; i.e., for $K_{i} \in \mathcal{K}^{n}$,

$$
K_{i} \rightarrow K \in \mathcal{K}^{n} \quad \Longrightarrow \quad S_{K_{i}} \rightarrow S_{K} \text {, weakly, as } i \rightarrow+\infty .
$$

For $K, L \in \mathcal{K}^{n}$, the mixed volume $V_{1}(K, L)$ may be defined by

$$
V_{1}(K, L)=\frac{1}{n} \int_{S^{n-1}} h_{L} d S_{K}
$$

In particular, for $K \in \mathcal{K}^{n}$,

$$
V_{1}(K, K)=V(K)
$$

or equivalently,

$$
V(K)=\frac{1}{n} \int_{S^{n-1}} h_{K} d S_{K} .
$$

Minkowski's inequality states that for convex bodies $K, L$ :

$$
V_{1}(K, L)^{n} \geq V(K)^{n-1} V(L)
$$


with equality if and only if $K$ and $L$ are homothetic.

We will use the following simple fact:

Let $f, f_{1}, \ldots \in C\left(S^{n-1}\right), \mu, \mu_{1}, \ldots$ be finite measures on $S^{n-1}$. If $f_{i} \rightarrow f$ uniformly on $S^{n-1}$, and $\mu_{i} \rightarrow \mu$ weakly on $S^{n-1}$, then

$$
\lim _{i \rightarrow+\infty} \int_{S^{n-1}} f_{i}(u) d \mu_{i}(u)=\int_{S^{n-1}} f(u) d \mu(u) .
$$

\section{Orlicz Norms}

Definition 3.1 [18] Let $\phi:[0, \infty) \rightarrow[0, \infty)$ be continuous, strictly increasing, continuously differentiable on $(0, \infty)$ with positive derivative, and satisfy $\lim _{t \rightarrow \infty} \phi(t)=\infty$. Let $\mu$ be a finite Borel measure on the sphere $S^{n-1}$. For a continuous function $f: S^{n-1} \rightarrow[0, \infty)$, the Orlicz norm $\|f\|_{\phi, \mu}$ is defined by

$$
\|f\|_{\phi, \mu}=\inf \left\{\lambda>0: \frac{1}{|\mu|} \int_{S^{n-1}} \phi\left(\frac{f}{\lambda}\right) d \mu \leq \phi(1)\right\} .
$$

As in [18], the Orlicz norm of a function $f$ not only depends on $\mu$ but also depends on $\phi$. The usual $L_{p}$ norm is obtained by taking $\phi(t)=t^{p}$.

As was shown in [18], the following properties for continuous $f: S^{n-1} \rightarrow[0, \infty)$ hold:

$$
\|c f\|_{\phi, \mu}=c\|f\|_{\phi, \mu}, \quad c>0 .
$$

In particular

$$
\|c\|_{\phi, \mu}=c, \quad c>0 .
$$

Moreover, the monotonicity of $\phi$ guarantees that for continuous $f, g: S^{n-1} \rightarrow$ $[0, \infty)$,

$$
f \leq g \quad \Longrightarrow \quad\|f\|_{\phi, \mu} \leq\|g\|_{\phi, \mu}
$$

Lemma 3.2 [18, Lemma 3] Suppose $\mu$ is a finite Borel measure on $S^{n-1}$ and the function $f: S^{n-1} \rightarrow[0, \infty)$ is continuous and such that $\mu(\{f \neq 0\})>0$. Then the Orlicz norm $\|f\|_{\phi, \mu}$ is positive and

$$
\|f\|_{\phi, \mu}=\lambda_{0} \Longleftrightarrow \frac{1}{|\mu|} \int_{S^{n-1}} \phi\left(\frac{f}{\lambda_{0}}\right) d \mu=\phi(1) .
$$

Lemma 3.3 [18, Lemma 4] Suppose $f: S^{n-1} \rightarrow[0, \infty)$ is a continuous function with $\mu(\{f \neq 0\})>0$ and $\left\{f_{i}\right\}$ is a sequence of nonnegative functions in $C\left(S^{n-1}\right)$ with $\mu\left(\left\{f_{i} \neq 0\right\}\right)>0$. If

$$
f_{i} \rightarrow f \quad \text { in } C\left(S^{n-1}\right)
$$

then

$$
\left\|f_{i}\right\|_{\phi, \mu} \rightarrow\|f\|_{\phi, \mu}
$$


Lemma 3.4 Suppose $f: S^{n-1} \rightarrow[0, \infty)$ is a continuous function with $\mu(\{f \neq 0\})$ $>0$ and $\left\{\mu_{i}\right\}$ is a sequence of finite Borel measures on $S^{n-1}$. If

$$
\mu_{i} \rightarrow \mu \text { weakly on } S^{n-1} \text {, }
$$

then

$$
\|f\|_{\phi, \mu_{i}} \rightarrow\|f\|_{\phi, \mu}
$$

Proof First, we will show that the sequence $\left\{\|f\|_{\phi, \mu_{i}}\right\}$ is bounded. The fact $f \in$ $C\left(S^{n-1}\right)$ guarantees that there exists a real $C>0$ such that $f(u) \leq C$ for all $u \in S^{n-1}$. Together with (18) and (19), it follows that

$$
0 \leq\|f\|_{\phi, \mu_{i}} \leq\|C\|_{\phi, \mu_{i}}=C
$$

for all Borel measures $\mu_{i}$ on $S^{n-1}, i \in \mathbb{N}$. Thus the boundedness of the sequence $\left\{\|f\|_{\phi, \mu_{i}}\right\}$ is established.

The Bolzano-Weierstrass theorem guarantees that the sequence $\left\{\|f\|_{\phi, \mu_{i}}\right\}$ has a convergent subsequence. In order to show that the sequence $\left\{\|f\|_{\phi, \mu_{i}}\right\}$ converges to $\|f\|_{\phi, \mu}$, it suffices to prove that every convergent subsequence converges to $\|f\|_{\phi, \mu}$. To simplify the notation, we denote an arbitrary convergent subsequence of $\left\{\|f\|_{\phi, \mu_{i}}\right\}$ by $\left\{\|f\|_{\phi, \mu_{i}}\right\}$ as well.

Next, we will show $\lim _{i \rightarrow \infty}\|f\|_{\phi, \mu_{i}}>0$. Suppose it is not true; i.e., $\|f\|_{\phi, \mu_{i}} \rightarrow 0$. The condition $\mu(\{f>0\})>0$ yields the result that there exists a sufficiently small $c>0$ such that $\mu(\{f \geq c\})>0$. Given $M>0$, there exists a sufficiently small $\delta>0$ such that $\phi\left(\frac{c}{\delta}\right)>\frac{M|\mu|}{\mu(\{f \geq c\})}$, since $\phi$ is strictly increasing and $\lim _{t \rightarrow \infty} \phi(t)=\infty$. Then the assumption $\lim _{i \rightarrow \infty}\|f\|_{\phi, \mu_{i}}=0$ implies there exists $N_{1}$ such that $\|f\|_{\phi, \mu_{i}}<\delta$ whenever $i>N_{1}$ for the above given $\delta$. Thus $\phi\left(\frac{f}{\|f\|_{\phi, \mu_{i}}}\right) \geq \phi\left(\frac{f}{\delta}\right)$ for $i>N_{1}$. Note that since $\mu(\{f \neq 0\})>0, f \in C\left(S^{n-1}\right)$ and $\mu_{i} \rightarrow \mu$ weakly on $S^{n-1}$, there exists $N_{2}$ such that $\mu_{i}(\{f \neq 0\})>0$ whenever $i>N_{2}$. From Lemma 3.2, it follows that

$$
\|f\|_{\phi, \mu_{i}}>0
$$

and

$$
\frac{1}{\left|\mu_{i}\right|} \int_{S^{n-1}} \phi\left(\frac{f}{\|f\|_{\phi, \mu_{i}}}\right) d \mu_{i}=\phi(1),
$$

whenever $i>N_{2}$.

We therefore deduce

$$
\begin{aligned}
\phi(1) & =\lim _{i \rightarrow \infty} \frac{1}{\left|\mu_{i}\right|} \int_{S^{n-1}} \phi\left(\frac{f}{\|f\|_{\phi, \mu_{i}}}\right) d \mu_{i} \\
& \geq \liminf _{i \rightarrow \infty} \frac{1}{\left|\mu_{i}\right|} \int_{S^{n-1}} \phi\left(\frac{f}{\delta}\right) d \mu_{i} \\
& =\frac{1}{|\mu|} \int_{S^{n-1}} \phi\left(\frac{f}{\delta}\right) d \mu \quad(\text { by (20)) }
\end{aligned}
$$




$$
\begin{aligned}
& \geq \frac{1}{|\mu|} \int_{\{f \geq c\}} \phi\left(\frac{f}{\delta}\right) d \mu \\
& \geq \phi\left(\frac{c}{\delta}\right) \frac{1}{|\mu|} \int_{\{f \geq c\}} d \mu \\
& >\frac{M|\mu|}{\mu(\{f \geq c\})} \cdot \frac{\mu(\{f \geq c\})}{|\mu|}=M,
\end{aligned}
$$

since $M>0$ is arbitrary, this is a contradiction.

Since $\lim _{i \rightarrow \infty}\|f\|_{\phi, \mu_{i}}>0$, by (22), there exists a real $c>0$ such that $\|f\|_{\phi, \mu_{i}}>c$ whenever $i>N_{2}$. Let $\lambda_{i}:=\|f\|_{\phi, \mu_{i}}$, then we may rewrite (23) as

$$
\int_{S^{n-1}} \phi\left(\frac{f}{\lambda_{i}}\right) d \overline{\mu_{i}}=\phi(1)
$$

where $d \overline{\mu_{i}}=d \mu_{i} /\left|\mu_{i}\right|$, for $i>N_{2}$.

Suppose that for the subsequence $\left\{\lambda_{i}\right\}$ we have $\lambda_{i} \rightarrow \lambda$.

Finally, we prove $\lambda=\|f\|_{\phi, \mu}$. Observe that

$$
\left|\frac{f(u)}{\lambda_{i}}-\frac{f(u)}{\lambda}\right|=\frac{f(u)\left|\lambda_{i}-\lambda\right|}{\lambda_{i} \lambda} \leq \frac{C\left|\lambda_{i}-\lambda\right|}{c^{2}} \quad \text { whenever } i>N_{2},
$$

which means that $f(u) / \lambda_{i}$ uniformly converges to $f(u) / \lambda$ for all $u \in S^{n-1}$. Note that $\left|f / \lambda_{i}\right| \leq C / c$ for $i>N_{2}$, and $\phi$ is uniformly continuous on $[0, C / c]$, hence the function $\phi\left(f(u) / \lambda_{i}\right)$ uniformly converges to $\phi(f(u) / \lambda)$ on $S^{n-1}$. Moreover, $\mu_{i} \rightarrow \mu$ weakly on $S^{n-1}$ implies $\bar{\mu}_{i} \rightarrow \bar{\mu}$ weakly on $S^{n-1}$, by (15), we obtain from (24) that

$$
\int_{S^{n-1}} \phi\left(\frac{f}{\lambda}\right) d \bar{\mu}=\phi(1) .
$$

Then, Lemma 3.2 again yields the desired result.

Corollary 3.5 Suppose $f: S^{n-1} \rightarrow[0, \infty)$ is a continuous function with $\mu(\{f \neq 0\})>0,\left\{f_{i}\right\}$ is a sequence of nonnegative functions in $C\left(S^{n-1}\right)$ and $\left\{\mu_{i}\right\}$ is a sequence of finite Borel measures on $S^{n-1}$. If

$$
f_{i} \rightarrow f \quad \text { in } C\left(S^{n-1}\right),
$$

and

$$
\mu_{i} \rightarrow \mu \text { weakly on } S^{n-1},
$$

then

$$
\left\|f_{i}\right\|_{\phi, \mu_{i}} \rightarrow\|f\|_{\phi, \mu}
$$

Proof Let $\varepsilon>0$ be given, by Lemma 3.3, there exists a sufficiently small $\delta>0$ such that

$$
\left|\|f+\delta\|_{\phi, \mu}-\|f\|_{\phi, \mu}\right|<\frac{\varepsilon}{2}
$$


and

$$
\left|\left\|f_{0}\right\|_{\phi, \mu}-\|f\|_{\phi, \mu}\right|<\frac{\varepsilon}{2},
$$

where $f_{0}:=\max \{f-\delta, 0\}$ satisfies $\mu\left(f_{0}>0\right)>0$.

Evidently, $f_{0}: S^{n-1} \rightarrow[0, \infty)$ is a continuous function. Since $f_{i} \rightarrow f \in C\left(S^{n-1}\right)$, there exists $N_{1}$ such that $f_{0} \leq f_{i} \leq f+\delta$ for $i \geq N_{1}$. Using (19), we obtain $\left\|f_{0}\right\|_{\phi, \mu_{i}} \leq\left\|f_{i}\right\|_{\phi, \mu_{i}} \leq\|f+\delta\|_{\phi, \mu_{i}}$ for $i \geq N_{1}$.

We now apply Lemma 3.4 to the function $f+\delta$ : there exists $N_{2}$ such that

$$
\left|\|f+\delta\|_{\phi, \mu_{i}}-\|f+\delta\|_{\phi, \mu}\right|<\frac{\varepsilon}{2}, \quad \text { whenever } i \geq N_{2} .
$$

Similarly, there exists $N_{3}$ such that

$$
\left|\left\|f_{0}\right\|_{\phi, \mu_{i}}-\left\|f_{0}\right\|_{\phi, \mu}\right|<\frac{\varepsilon}{2} \quad \text { whenever } i \geq N_{3} \text {. }
$$

For $N=\max \left\{N_{1}, N_{2}, N_{3}\right\}$, the inequalities (25) and (27) yield

$$
\left|\|f+\delta\|_{\phi, \mu_{i}}-\|f\|_{\phi, \mu}\right|<\varepsilon \quad \text { whenever } i \geq N .
$$

Similarly, the inequalities (26) and (28) yield

$$
\left|\left\|f_{0}\right\|_{\phi, \mu_{i}}-\|f\|_{\phi, \mu}\right|<\varepsilon \quad \text { whenever } i \geq N \text {. }
$$

Since $\left\|f_{0}\right\|_{\phi, \mu_{i}} \leq\left\|f_{i}\right\|_{\phi, \mu_{i}} \leq\|f+\delta\|_{\phi, \mu_{i}}$, the two inequalities above give

$$
\left|\left\|f_{i}\right\|_{\phi, \mu_{i}}-\|f\|_{\phi, \mu}\right|<\varepsilon
$$

whenever $i>N$.

Recall (6) that $h_{\hat{v}}(u)=(u \cdot v)_{+}=\frac{|u \cdot v|+u \cdot v}{2}$.

Lemma 3.6 If $\mu$ is a finite Borel measure on the sphere $S^{n-1}$ whose support is not contained in a closed hemisphere, then there exists a real $c>0$ such that $\left\|h_{\hat{v}}\right\|_{\phi, \mu} \geq c$ for every $v \in S^{n-1}$.

Proof Since the support of $\mu$ is not contained in a closed hemisphere of $S^{n-1}$

$$
\mu\left(\left\{h_{\hat{v}}>0\right\}\right)=\mu\left(S^{n-1} \backslash H_{v, 0}^{-}\right)>0 \quad \text { for every } v \in S^{n-1} \text {. }
$$

According to Lemma 3.2, we have $\left\|h_{\hat{v}}\right\|_{\phi, \mu}>0$. Since $S^{n-1}$ is compact, it suffices to show that the function $v \mapsto\left\|h_{\hat{v}}\right\|_{\phi, \mu}$ is continuous.

Suppose $v_{i} \in S^{n-1}$ and $v_{i} \rightarrow v$ as $i \rightarrow+\infty$. Note that $h_{\hat{v}}(u)=\frac{|u \cdot v|+u \cdot v}{2}$ implies $h_{\hat{v}_{i}} \rightarrow h_{\hat{v}}$ uniformly on $S^{n-1}$, and thus $\left\|h_{\hat{v}_{i}}\right\|_{\phi, \mu}$ converges to $\left\|h_{\hat{v}}\right\|_{\phi, \mu}$ by Lemma 3.3. So the desired continuity of $v \mapsto\left\|h_{\hat{v}}\right\|_{\phi, \mu}$ is established. 
Corollary 3.7 If a sequence of finite discrete measures $\left\{\mu_{i}\right\}$ weakly converges to a finite Borel measure $\mu$ on $S^{n-1}$, and the supports of $\mu_{i}$ and $\mu$ are all not contained in a closed hemisphere, $i \in \mathbb{N}$. Then, there exists a real $c>0$ such that $\left\|h_{\hat{v}}\right\|_{\phi, \mu_{i}} \geq c$ for every $v \in S^{n-1}$ and $i \in \mathbb{N}$.

Proof Recall that a finite discrete measure $\mu_{i}$ on $S^{n-1}$ is a Borel measure. Observe that $h_{\hat{v}} \leq 1$, combining (19) and (18), we have $\left\|h_{\hat{v}}\right\|_{\phi, \mu} \leq 1$ for any Borel measure $\mu$. From Lemma 3.6, for each $i$, let

$$
c_{i}=\min _{v \in S^{n-1}}\left\|h_{\hat{v}}\right\|_{\phi, \mu_{i}}=\left\|h_{\widehat{v_{i}}}\right\|_{\phi, \mu_{i}}>0
$$

where $v_{i} \in S^{n-1}$ is any point where this minimum is attained. Similarly, there exists $c_{0}>0$ such that $\left\|h_{\hat{v}}\right\|_{\phi, \mu} \geq c_{0}$ for all $v \in S^{n-1}$. The compactness of $[0,1]$ guarantees that the sequence $\left\{c_{i}\right\}$ has a convergent subsequence.

In order to prove this corollary, it suffices to show that no convergent subsequence of the sequence $\left\{c_{i}\right\}$ converges to 0 . We argue by contradiction, assume there exists a subsequence $\left\{c_{i^{\prime}}\right\}$ of the sequence $\left\{c_{i}\right\}$ such that $c_{i^{\prime}} \rightarrow 0$. From (29), for the subsequence $\left\{c_{i^{\prime}}\right\}$ we can write $c_{i^{\prime}}=\left\|h_{\widehat{v_{i^{\prime}}}}\right\|_{\phi, \mu_{i^{\prime}}}$ for some $v_{i^{\prime}} \in S^{n-1}$ as well. Since $S^{n-1}$ is compact, there exists a subsequence $\left\{v_{i^{\prime \prime}}\right\}$ of the sequence $\left\{v_{i^{\prime}}\right\}$ such that $v_{i^{\prime \prime}}$ converges to $v_{0} \in S^{n-1}$. Thus we obtain a subsequence $\left\{c_{i^{\prime \prime}}\right\}$ of the sequence $\left\{c_{i^{\prime}}\right\}$ such that $c_{i^{\prime \prime}}$ converges to 0 by the assumption, where $c_{i^{\prime \prime}}=\left\|h_{\widehat{v_{i^{\prime \prime}}}}\right\|_{\phi, \mu_{i^{\prime \prime}}}$. Meanwhile, $h_{\widehat{v_{i^{\prime \prime}}}}$ converges to $h_{\hat{v}}$ uniformly on $S^{n-1}$ as shown in Lemma 3.6. By Corollary 3.5, we see that $c_{i^{\prime \prime}}=\left\|h_{\widehat{v_{i^{\prime \prime}}}}\right\|_{\phi, \mu_{i^{\prime \prime}}} \rightarrow\left\|h_{\widehat{v_{0}}}\right\|_{\phi, \mu}$. Note that $\left\|h_{\hat{v}}\right\|_{\phi, \mu} \geq c_{0}$ for all $v \in S^{n-1}$, while $c_{i^{\prime \prime}}=\left\|h_{\widehat{v_{i^{\prime \prime}}}}\right\|_{\phi, \mu_{i^{\prime \prime}}} \rightarrow 0$, this is the desired contradiction.

\section{The Orlicz Minkowski Problem for Discrete Measures}

This section is devoted to the proof of Theorem 1.1.

Lemma 4.1 Suppose $\varphi:(0, \infty) \rightarrow(0, \infty)$ is a continuous function such that $\phi(t)=$ $\int_{o}^{t} \frac{1}{\varphi(s)} d s$ exists for every positive $t$ and is unbounded as $t \rightarrow \infty$, and $\varphi(s)$ tends to infinity as $s \rightarrow 0^{+}$. Then,

(i) $\lim _{t \rightarrow 0^{+}} \phi(t)=0, \lim _{t \rightarrow 0^{+}} \phi^{\prime}(t)=0$.

(ii) Let $\alpha(t):=\frac{1}{\varphi(t)}, \beta(t):=\frac{t}{\varphi(t)}$, both of them are continuous on $(0, \infty)$. Moreover, we can extend the domain of $\alpha(t), \beta(t)$ right continuously to the origin; i.e., $\alpha(0)=\lim _{t \rightarrow 0^{+}} \alpha(t)=0, \beta(0)=\lim _{t \rightarrow 0^{+}} \beta(t)=0$.

(iii) $\lim _{t \rightarrow 0^{+}}\left(\phi^{-1}\right)^{\prime}(\phi(h)-b \phi(t))$ is bounded from above and below by positive reals, for any given $b, h>0$.

Proof First, we extend the domain of $\phi$ to $[0, \infty)$ by

$$
\phi(t)=\int_{o}^{t} \frac{1}{\varphi(s)} d s, \quad \text { for } t>0, \quad \text { and } \quad \phi(0)=\lim _{t \rightarrow 0^{+}} \phi(t) .
$$

Indeed, (i) and (ii) are easily verified. 
Next, we will show (iii).

Note that the function $\phi$ is strictly increasing and continuously differentiable on $(0, \infty)$, and $\phi^{\prime}>0$. Since $\lim _{t \rightarrow 0^{+}} \phi(t)=0$ and $\lim _{t \rightarrow \infty} \phi(t)=\infty, \phi$ has an inverse $\phi^{-1}:[0, \infty) \rightarrow[0, \infty)$ which is continuously differentiable on $(0, \infty)$.

Hence for any given $b, h>0$, there exists $\delta>0$ such that

$$
\phi(h)-b \phi(t) \in \phi\left(\left(\frac{h}{2}, h\right)\right)
$$

for all $t \in(0, \delta)$. Obviously, $\phi\left(\left(\frac{h}{2}, h\right)\right) \subset(0, \infty)$, thus $\left(\phi^{-1}\right)^{\prime}(\phi(h)-b \phi(t))$ exists for a sufficiently small $t$. Observe $\left(\phi^{-1}\right)^{\prime}(\phi(s))=\frac{1}{\phi^{\prime}(s)}=\varphi(s)$ when $s \in\left(\frac{h}{2}, h\right)$, and the function $\varphi$ is continuous on $(0, \infty)$, thus $\lim _{t \rightarrow 0^{+}}\left(\phi^{-1}\right)^{\prime}(\phi(h)-b \phi(t))$ is bounded from above and below by positive reals.

By this lemma we can assume in the proofs of Theorems 1.1 and 1.2 that the domains of $\phi(t), \alpha(t)$, and $\beta(t)$ contain zero; i.e., $\phi(0)=\lim _{t \rightarrow 0^{+}} \phi(t)=0, \alpha(0)=$ $\lim _{t \rightarrow 0^{+}} \alpha(t)=0$, and $\beta(0)=\lim _{t \rightarrow 0^{+}} \beta(t)=0$.

In the following, we denote by $\mathbb{R}_{+}^{m}$ the set of all $x=\left(x_{1}, \ldots, x_{m}\right) \in \mathbb{R}^{m}$ with positive components. Recall that $H_{u, t}^{-}:=\left\{y \in \mathbb{R}^{n}: u \cdot y \leq t\right\}$ is the halfspace with exterior normal vector $u$ and the distance $t \geq 0$ from the origin.

Lemma 4.2 [20, Lemma 3.2] Let $u_{1}, \ldots, u_{m} \in S^{n-1}$ be pairwise distinct vectors which are not contained in a closed hemisphere. For $x \in \mathbb{R}_{+}^{m}$, let $P(x):=$ $\bigcap_{i=1}^{m} H_{u_{i}, x_{i}}^{-}$. Then $V(P(x))$ is of class $C^{1}$ and $\partial_{i} V(P(x))=S\left(P(x),\left\{u_{i}\right\}\right)$ for $i=1, \ldots, m$.

With these lemmas in hand, we can complete the proof of Theorem 1.1.

Proof of Theorem 1.1 Let $\mathbb{R}_{*}^{m}$ be the set of all $x=\left(x_{1}, \ldots, x_{m}\right) \in \mathbb{R}^{m}$ with nonnegative components, define the set

$$
M:=\left\{x \in \mathbb{R}_{*}^{m}: \sum_{i=1}^{m} \alpha_{i} \phi\left(x_{i}\right)=\sum_{i=1}^{m} \alpha_{i} \phi(1)\right\} .
$$

Since $\phi$ is strictly increasing, the surface $M$ is compact. For $x \in M$, we define $P(x)$ as the convex polytope

$$
P(x):=\bigcap_{i=1}^{m} H_{u_{i}, x_{i}}^{-}=\bigcap_{i=1}^{m} H_{u_{i}, h_{i}^{x}}^{-},
$$

where $h_{i}^{x}:=h\left(P(x), u_{i}\right)$ for $i=1, \ldots, m$.

Since $M$ is compact and the function $x \mapsto V(P(x))$ is continuous, there is a point $z \in M$ such that

$$
V(P(x)) \leq V(P(z)) \quad \text { for all } x \in M
$$


Note that if we set $x^{*}=(1, \ldots, 1) \in \mathbb{R}^{m}$, then clearly $x^{*} \in M$. Since $0<V\left(B_{2}^{n}\right) \leq$ $V\left(P\left(x^{*}\right)\right) \leq V(P(z))$, this gives $P(z) \in \mathcal{P}^{n}$.

We will prove that $P(z)$ is the desired polytope.

First, we want to show

$$
0 \in \operatorname{int}(P(z))
$$

Observe for any $x \in M, 0 \in P(x)$, that is, either $0 \in \operatorname{bd}(P(z))$ or $0 \in \operatorname{int}(P(z))$. Recall that $h_{i}^{z}:=h\left(P(z), u_{i}\right)$ for $i=1, \ldots, m$. Suppose $0 \in \operatorname{bd}(P(z))$, so there exist $h_{1}^{z}=$ $\ldots=h_{k}^{z}=0$ and $h_{k+1}^{z}, \ldots, h_{m}^{z}>0$ for some $1 \leq k<m$. We shall get a contradiction by showing that under this assumption there is some $z_{t} \in M$ such that $V\left(P\left(z_{t}\right)\right)>$ $V(P(z))$, which contradicts the definition of $z$. For a small $t>0$, we define

$$
\begin{aligned}
z_{t}:= & \left(\phi^{-1}\left(\phi\left(z_{1}\right)+\phi(t)\right), \ldots, \phi^{-1}\left(\phi\left(z_{k}\right)+\phi(t)\right),\right. \\
& \left.\phi^{-1}\left(\phi\left(z_{k+1}\right)-\alpha \phi(t)\right), \ldots, \phi^{-1}\left(\phi\left(z_{m}\right)-\alpha \phi(t)\right)\right),
\end{aligned}
$$

where

$$
\alpha:=\frac{\sum_{i=1}^{k} \alpha_{i}}{\sum_{i=k+1}^{m} \alpha_{i}} .
$$

Obviously, $z_{t} \in M$ if $t>0$ is sufficiently small.

Analogously, for a small $t>0$, we define

$$
\begin{aligned}
h_{t}^{z}:= & \left(\phi^{-1}\left(\phi\left(h_{1}^{z}\right)+\phi(t)\right), \ldots, \phi^{-1}\left(\phi\left(h_{k}^{z}\right)+\phi(t)\right),\right. \\
& \left.\phi^{-1}\left(\phi\left(h_{k+1}^{z}\right)-\alpha \phi(t)\right), \ldots, \phi^{-1}\left(\phi\left(h_{m}^{z}\right)-\alpha \phi(t)\right)\right) .
\end{aligned}
$$

Since $\phi(0)=0$, by (30), we have

$$
P\left(h_{t}^{z}\right):=\bigcap_{i=1}^{k} H_{u_{i}, t}^{-} \cap \bigcap_{i=k+1}^{m} H_{u_{i}, \phi^{-1}\left(\phi\left(h_{i}^{z}\right)-\alpha \phi(t)\right)}^{-} .
$$

A glance at (30) again shows $P\left(h_{0}^{z}\right)=P(z)$. Since $h_{i}^{z} \leq z_{i}$ for all $i \leq m$ and the function $\phi$ is strictly increasing, $P\left(h_{t}^{z}\right) \subset P\left(z_{t}\right)$ and $0 \in \operatorname{int}\left(P\left(h_{t}^{z}\right)\right)$, if $t>0$ is sufficiently small. For simplicity, we write

$$
S_{i}:=S\left(P(z), u_{i}\right) \quad \text { and } \quad S_{i}^{t}:=S\left(P\left(h_{t}^{z}\right), u_{i}\right),
$$

and thus for the $n$-dimensional polytope $P\left(h_{t}^{z}\right), P(z)$, according to the discrete form of (13) and (12), it follows that

$$
n V\left(P\left(h_{t}^{z}\right)\right)=t \sum_{i=1}^{k} S_{i}^{t}+\sum_{i=k+1}^{m} \phi^{-1}\left(\phi\left(h_{i}^{z}\right)-\alpha \phi(t)\right) S_{i}^{t},
$$

and

$$
n V_{1}\left(P\left(h_{t}^{z}\right), P(z)\right)=0 \sum_{i=1}^{k} S_{i}^{t}+\sum_{i=k+1}^{m} h_{i}^{z} S_{i}^{t}
$$


Note that each open set intersecting $P(z)$ intersects $P\left(h_{t}^{z}\right)$ if $t>0$ is sufficiently small and each closed set having empty intersection with $P(z)$ has empty intersection with $P\left(h_{t}^{z}\right)$ if $t>0$ is sufficiently small. Hence $P\left(h_{t}^{z}\right) \rightarrow P(z)$ as $t \rightarrow 0^{+}$[49, p. 57], and (11) gives $S_{i}^{t} \rightarrow S_{i}$ as $t \rightarrow 0^{+}$. Now, we conclude that

$$
\begin{aligned}
\lim _{t \rightarrow 0^{+}} & \frac{V\left(P\left(h_{t}^{z}\right)\right)-V_{1}\left(P\left(h_{t}^{z}\right), P(z)\right)}{t} \\
= & \frac{1}{n} \lim _{t \rightarrow 0^{+}}\left(\sum_{i=1}^{k} \frac{t-0}{t} S_{i}^{t}+\sum_{i=k+1}^{m} \frac{\phi^{-1}\left(\phi\left(h_{i}^{z}\right)-\alpha \phi(t)\right)-h_{i}^{z}}{t} S_{i}^{t}\right) \\
& =\frac{1}{n} \sum_{i=1}^{k} S_{i}>0 .
\end{aligned}
$$

Since $\lim _{t \rightarrow 0^{+}} \phi^{\prime}(t)=0$ and $\lim _{t \rightarrow 0^{+}}\left(\phi^{-1}\right)^{\prime}\left(\phi\left(h_{i}^{z}\right)-\alpha \phi(t)\right)$ is bounded by Lemma 4.1

$$
\lim _{t \rightarrow 0^{+}} \frac{\phi^{-1}\left(\phi\left(h_{i}^{z}\right)-\alpha \phi(t)\right)-h_{i}^{z}}{t}=\lim _{t \rightarrow 0^{+}}\left(\phi^{-1}\right)^{\prime}\left(\phi\left(h_{i}^{z}\right)-\alpha \phi(t)\right)\left(-\alpha \phi^{\prime}(t)\right)=0,
$$

thus the last equality is obtained. By Minkowski's inequality (14) and $P\left(h_{t}^{z}\right) \rightarrow P(z)$ as $t \rightarrow 0^{+}$, we obtain

$$
\begin{aligned}
0 & <\lim _{t \rightarrow 0^{+}} \frac{V\left(P\left(h_{t}^{z}\right)\right)-V_{1}\left(P\left(h_{t}^{z}\right), P(z)\right)}{t} \\
& \leq \liminf _{t \rightarrow 0^{+}} \frac{V\left(P\left(h_{t}^{z}\right)\right)-V\left(P\left(h_{t}^{z}\right)\right)^{1-\frac{1}{n}} V(P(z))^{\frac{1}{n}}}{t} \\
& =V(P(z))^{1-\frac{1}{n}} \liminf _{t \rightarrow 0^{+}} \frac{V\left(P\left(h_{t}^{z}\right)\right)^{\frac{1}{n}}-V(P(z))^{\frac{1}{n}}}{t} .
\end{aligned}
$$

Consequently, $V\left(P\left(h_{t}^{z}\right)\right)>V(P(z))$ if $t>0$ is sufficiently small. However, since $P\left(h_{t}^{z}\right) \subset P\left(z_{t}\right)$, the required contradiction $V\left(P\left(z_{t}\right)\right)>V(P(z))$ follows.

Next, we will prove the main conclusions (1) and (2). From (31), we have

$$
z \in M_{+}:=\left\{x \in \mathbb{R}_{+}^{m}: \sum_{i=1}^{m} \alpha_{i} \phi\left(x_{i}\right)=\sum_{i=1}^{m} \alpha_{i} \phi(1)\right\} .
$$

By the Lagrange multiplier rule there is some $\lambda \in \mathbb{R}$ such that

$$
\nabla V(P(z))=\lambda \nabla\left(\sum_{i=1}^{m} \alpha_{i} \phi\left(z_{i}\right)-\sum_{i=1}^{m} \alpha_{i} \phi(1)\right)
$$

where $V(P(z))$ is differentiable by Lemma 4.2, and $\phi^{\prime}\left(z_{i}\right)$ exists since $z_{i}>0$ for all $i=1, \ldots, m$. Thus

$$
S_{i}=\lambda \frac{\alpha_{i}}{\varphi\left(z_{i}\right)}, \quad i=1, \ldots, m
$$


The fact that $S_{i}>0$ for some $i \in\{1, \ldots, m\}$ and $\alpha_{i}, \varphi\left(z_{i}\right)>0$ for all $i=1, \ldots, m$, shows $\lambda>0$. Together with the above expression (32), it follows that $S_{i}>0$ for all $i=1, \ldots, m$. Hence, $h\left(P(z), u_{i}\right)=z_{i}$ for all $i=1, \ldots, m$. By the discrete form of (13), and (32), it follows that

$$
n V(P(z))=\sum_{i=1}^{m} S_{i} z_{i}=\lambda \sum_{i=1}^{m} \frac{\alpha_{i} z_{i}}{\varphi\left(z_{i}\right)} .
$$

Therefore, for $i=1, \ldots, m$,

$$
S\left(P(z), u_{i}\right)=S_{i}=\frac{\alpha_{i}}{c \varphi\left(z_{i}\right)}
$$

where $c=\frac{1}{n V(P(z))} \sum_{i=1}^{m} \frac{\alpha_{i} z_{i}}{\varphi\left(z_{i}\right)}$.

Indeed, together with $h\left(P(z), u_{i}\right)=z_{i}$, it follows that

$$
\mu=\sum_{i=1}^{m} \alpha_{i} \delta_{u_{i}}=c \varphi(h(P(z), \cdot)) S(P(z), \cdot) .
$$

Observe the definition of $M_{+}$and $h\left(P(z), u_{i}\right)=z_{i}$, hence

$$
\sum_{i=1}^{m} \alpha_{i} \phi\left(h\left(P(z), u_{i}\right)\right)=\sum_{i=1}^{m} \alpha_{i} \phi(1) .
$$

Moreover, take $\mu=\sum_{i=1}^{m} \alpha_{i} \delta_{u_{i}}$ in Lemma 3.2 to conclude

$$
\left\|h_{P}(z)\right\|_{\phi, \mu}=1
$$

\section{The Orlicz Minkowski Problem for General Measures}

Proof of Theorem 1.2 As was shown in [49, Theorem 7.1.2], for a given Borel measure $\mu$ on $S^{n-1}$ which is not concentrated in a closed hemisphere, one can construct a sequence of discrete measures $\left\{\mu_{i}\right\}$ on $S^{n-1}, i \in \mathbb{N}$, such that the support of $\mu_{i}$ is not contained in a closed hemisphere and $\mu_{i} \rightarrow \mu$ weakly as $i \rightarrow \infty$. By Theorem 1.1, for each $i \in \mathbb{N}$ there exists a polytope $P_{i} \in \mathcal{P}_{0}^{n}$ with

$$
\mu_{i}=c_{i} \varphi\left(h\left(P_{i}, \cdot\right)\right) S\left(P_{i}, \cdot\right) .
$$

First, we claim that the sequence $\left\{P_{i}\right\}$ is bounded. For each $i$, let $v_{i} \in S^{n-1}$ be chosen such that $r_{i} v_{i} \in P_{i}$ with $\left|r_{i} v_{i}\right|$ maximal for suitable $r_{i}>0$. Thus we derive $r_{i} h_{\hat{v}_{i}}(u) \leq h_{P_{i}}(u)$ for all $u \in S^{n-1}$ from (8) and (7). Together with (17), (19), and (2), we see that

$$
r_{i}\left\|h_{\hat{v}_{i}}\right\|_{\phi, \mu_{i}}=\left\|r_{i} h_{\hat{v}_{i}}\right\|_{\phi, \mu_{i}} \leq\left\|h_{P_{i}}\right\|_{\phi, \mu_{i}}=1 \text {. }
$$


By Corollary 3.7, there exists a real $c>0$ such that $\left\|h_{\hat{v}}\right\|_{\phi, \mu_{i}} \geq c$ for every $v \in S^{n-1}$, $i \in \mathbb{N}$. It follows that the $r_{i}$ 's are bounded from above, and hence the sequence $\left\{P_{i}\right\}$ is bounded. We set $h_{P_{i}} \leq R$ for $i \in \mathbb{N}$.

Now Blaschke's selection theorem guarantees the existence of a convergent subsequence of $\left\{P_{i}\right\}$, which will also be denoted by $\left\{P_{i}\right\}$, with $\lim _{i \rightarrow \infty} P_{i}=K$. Now, $0<V\left(B_{2}^{n}\right) \leq V\left(P_{i}\right)$ for all $i \in \mathbb{N}$ implies that $0<V\left(B_{2}^{n}\right) \leq V(K)$, that is, $K \in \mathcal{K}^{n}$. Thus, from (9) it is clear that $h\left(P_{i}, \cdot\right) \rightarrow h(K, \cdot)$ uniformly on $S^{n-1}$. Since $0 \in \operatorname{int}\left(P_{i}\right)$ for all $i \in \mathbb{N}$, this gives $0 \in K$.

For a discrete measure $\mu_{i}$, we rewrite $c_{i}=\frac{1}{n V\left(P_{i}\right)} \int_{S^{n-1}} \frac{h_{P_{i}}}{\varphi\left(h_{P_{i}}\right)} d \mu_{i}$. We also set $c=\frac{1}{n V(K)} \int_{S^{n-1}} \frac{h_{K}}{\varphi\left(h_{K}\right)} d \mu$. From Lemma 4.1(ii) and $0<h_{P_{i}} \leq R$, we find that $\beta(t):=\frac{t}{\varphi(t)}$ is uniformly continuous on $[0, R]$. Meanwhile, $h\left(P_{i}, \cdot\right) \rightarrow h(K, \cdot)$ uniformly on $S^{n-1}$, and it follows that $\frac{h\left(P_{i}, \cdot\right)}{\varphi\left(h\left(P_{i}, \cdot\right)\right)}$ uniformly converges to $\frac{h(K, \cdot)}{\varphi(h(K, \cdot))}$ on $S^{n-1}$. Combining $V\left(P_{i}\right) \rightarrow V(K)$ and $\mu_{i} \rightarrow \mu$ weakly, as $i \rightarrow \infty$, by (15), we obtain $c_{i} \rightarrow c$ as $i \rightarrow \infty$.

Next, for a continuous function $f \in C\left(S^{n-1}\right)$ and $i \in \mathbb{N}$, we have

$$
\int_{S^{n-1}} \frac{f(u)}{\varphi\left(h\left(P_{i}, u\right)\right)} d \mu_{i}(u)=c_{i} \int_{S^{n-1}} f(u) d S\left(P_{i}, u\right) .
$$

From Lemma 4.1(ii) and $0<h_{P_{i}} \leq R$, we see that $\alpha(t):=\frac{1}{\varphi(t)}$ is uniformly continuous on $[0, R]$. Meanwhile, $h\left(P_{i}, \cdot\right) \rightarrow h(K, \cdot)$ uniformly on $S^{n-1}$, and it follows that $\frac{1}{\varphi\left(h\left(P_{i}, \cdot\right)\right)}$ uniformly converges to $\frac{1}{\varphi(h(K, \cdot))}$ on $S^{n-1}$. Since $\mu_{i} \rightarrow \mu$ and $S\left(P_{i}, \cdot\right) \rightarrow S(K, \cdot)$ weakly by (11), as $i \rightarrow \infty$, using (15) again, it follows from (34) that

$$
\int_{S^{n-1}} \frac{f(u)}{\varphi(h(K, u))} d \mu(u)=c \int_{S^{n-1}} f(u) d S(K, u) .
$$

The existence assertion (3) now follows, since (35) holds for any $f \in C\left(S^{n-1}\right)$.

Moreover, since $\left\|h_{P_{i}}\right\|_{\phi, \mu_{i}}=1$, and $h\left(P_{i}, \cdot\right) \rightarrow h(K, \cdot)$ uniformly on $S^{n-1}$, we obtain from Corollary 3.5 that

$$
\left\|h_{K}\right\|_{\phi, \mu}=1
$$

The following proof of Corollary 5.1 is similar to the one of [18, Corollary 2], which is known as the $L_{p}$-Minkowski problem. We include it for the sake of completeness.

Corollary 5.1 If $\mu$ is a finite Borel measure on $S^{n-1}$ which is not concentrated on a closed hemisphere, then

(i) for $p>1$, there exists a convex body $K \in \mathcal{K}^{n}$ with $0 \in K$ such that

$$
h_{K}^{p-1} d \mu=c d S_{K},
$$

where $c=1 / V(K)$. 
(ii) for $1<p \neq n$, there exists a convex body $K \in \mathcal{K}^{n}$ with $0 \in K$ such that

$$
h_{K}^{p-1} d \mu=d S_{K}
$$

Proof (i) Note that $\varphi(t)=t^{1-p}$ for $p>1$ satisfies the requirements of Theorem 1.2. From (3) we have (36) and from (4) it follows that

$$
\frac{1}{|\mu|} \int_{S^{n-1}} h_{K}^{p} d \mu=1 .
$$

Using (13) and (3), we conclude

$$
\int_{S^{n-1}} h_{K}^{p} d \mu=\operatorname{cn} V(K) .
$$

From (38) and (39) it follows that $c=|\mu| / n V(K)$. Therefore

$$
h_{K}^{p-1} d \mu=\frac{|\mu|}{n V(K)} d S_{K} .
$$

In order to get the desired $c=1 / V(K)$, we will show that a dilation of $K$ yields this $c$. Let $K=\lambda K^{\prime}$, then the homogeneity properties of (8) and (10) imply

$$
\lambda^{p} h_{K^{\prime}}^{p-1} d \mu=\frac{|\mu|}{n V\left(K^{\prime}\right)} d S_{K^{\prime}} .
$$

Then $K^{\prime}$ is the desired convex body, by choosing $\lambda^{p}=|\mu| / n$.

(ii) If $K^{\prime \prime}=\lambda K$ satisfies (37), then by (8) and (10) we have

$$
h_{K}^{p-1} d \mu=\lambda^{n-p} d S_{K}
$$

Since there exists $K$ satisfying (36), we can choose $\lambda^{n-p}=c$, where $n \neq p$ makes the reverse process feasible.

Acknowledgements Supported in part by the National Natural Science Foundation of China (Grant No. 11071156) and Shanghai Leading Academic Discipline Project (Project Number: J50101).

The authors are grateful to the anonymous referees for their careful reading and many valuable suggestions, such that the paper is greatly improved.

\section{References}

1. Aleksandrov, A.D.: On the theory of mixed volumes. III. Extension of two theorems of Minkowski on convex polyhedra to arbitrary convex bodies. Mat. Sb. 3, 27-46 (1938) (in Russian)

2. Aleksandrov, A.D.: On the surface area measure of convex bodies. Mat. Sb. 6, 167-174 (1939) (in Russian)

3. Aleksandrov, A.D.: Smoothness of the convex surface of bounded Gaussian curvature. Dokl. Akad. Nauk SSSR 36, 195-199 (1942)

4. Campi, S., Gronchi, P.: The $L^{p}$-Busemann-Petty centroid inequality. Adv. Math. 167, 128-141 (2002)

5. Campi, S., Gronchi, P.: On the reverse $L^{p}$-Busemann-Petty centroid inequality. Mathematika 49, $1-11$ (2002) 
6. Chen, W.: $L_{p}$ Minkowski problem with not necessarily positive data. Adv. Math. 201, 77-89 (2006)

7. Chou, K.-S., Wang, X.-J.: A logarithmic Gauss curvature flow and the Minkowski problem. Ann. Inst. Henri Poincaré, Anal. Non Linéaire 17(6), 733-751 (2000)

8. Chou, K.-S., Wang, X.-J.: The $L_{p}$-Minkowski problem and the Minkowski problem in centroaffine geometry. Adv. Math. 205, 33-83 (2006)

9. Cianchi, A., Lutwak, E., Yang, D., Zhang, G.: Affine Moser-Trudinger and Morrey-Sobolev inequalities. Calc. Var. Partial Differ. Equ. 36, 419-436 (2009)

10. Fenchel, W., Jessen, B.: Mengenfunktionen und konvexe Körper. Danske Vid. Selskab. Mat.-fys. Medd. 16, 1-31 (1938)

11. Gardner, R.J.: Geometric Tomography, 2nd edn. Encyclopedia of Mathematics and Its Applications, vol. 58. Cambridge University Press, Cambridge (2006)

12. Gruber, P.M.: Convex and Discrete Geometry. Grundlehren Math. Wiss., vol. 336. Springer, Berlin (2007)

13. Guan, P., Lin, C.-S.: On equation $\operatorname{det}\left(u_{i j}+\delta_{i j} u\right)=u^{p} f$ on $S^{n}$. Preprint No. 2000-7, NCTS in TsingHua University (2000)

14. Haberl, C.: $L_{p}$ intersection bodies. Adv. Math. 217, 2599-2624 (2008)

15. Haberl, C.: Star body valued valuations. Indiana Univ. Math. J. 58, 2253-2276 (2009)

16. Haberl, C., Schuster, F.: General $L_{p}$ affine isoperimetric inequalities. J. Differ. Geom. 83, 1-26 (2009)

17. Haberl, C., Schuster, F.: Asymmetric affine $L_{p}$ Sobolev inequalities. J. Funct. Anal. 257, 641-658 (2009)

18. Haberl, C., Lutwak, E., Yang, D., Zhang, G.: The even Orlicz Minkowski problem. Adv. Math. 224, 2485-2510 (2010)

19. Hu, C., Ma, X.-N., Shen, C.: On the Christoffel-Minkowski problem of Firey's p-sum. Calc. Var. Partial Differ. Equ. 21, 137-155 (2004)

20. Hug, D., Lutwak, E., Yang, D., Zhang, G.: On the $L_{p}$ Minkowski problem for polytopes. Discrete Comput. Geom. 33, 699-715 (2005)

21. Jerison, D.: A Minkowski problem for electrostatic capacity. Acta Math. 176, 1-47 (1996)

22. Jiang, M.-Y.: Remarks on the 2-dimensional $L_{p}$-Minkowski problem. Adv. Nonlinear Stud. 10, 297313 (2010)

23. Klain, D.: The Minkowski problem for polytopes. Adv. Math. 185, 270-288 (2004)

24. Lamberg, L.: On the Minkowski problem and the lightcurve operator. Ann. Acad. Sci. Fenn., Ser. A 1 Math. 87, 1-107 (1993)

25. Lamberg, L., Kaasalainen, M.: Numerical solution of the Minkowski problem. J. Comput. Appl. Math. 137(2), 213-227 (2001)

26. Ludwig, M.: Ellipsoids and matrix-valued valuations. Duke Math. J. 119, 159-188 (2003)

27. Ludwig, M.: Minkowski valuations. Trans. Am. Math. Soc. 357, 4191-4213 (2005)

28. Ludwig, M.: General affine surface areas. Adv. Math. 224, 2346-2360 (2010)

29. Ludwig, M., Reitzner, M.: A classification of SL(n) invariant valuations. Ann. Math. 172(2), 1223$1271(2010)$

30. Lutwak, E.: The Brunn-Minkowski-Firey theory. I. Mixed volumes and the Minkowski problem. J. Differ. Geom. 38, 131-150 (1993)

31. Lutwak, E., Oliker, V.: On the regularity of solutions to a generalization of the Minkowski problem. J. Differ. Geom. 41, 227-246 (1995)

32. Lutwak, E.: The Brunn-Minkowski-Firey theory. II. Affine and geominimal surface areas. Adv. Math. 118, 244-294 (1996)

33. Lutwak, E., Yang, D., Zhang, G.: $L_{p}$ affine isoperimetric inequalities. J. Differ. Geom. 56, 111-132 (2000)

34. Lutwak, E., Yang, D., Zhang, G.: A new ellipsoid associated with convex bodies. Duke Math. J. 104, 375-390 (2000)

35. Lutwak, E., Yang, D., Zhang, G.: Sharp affine $L_{p}$ Sobolev inequalities. J. Differ. Geom. 62, 17-38 (2002)

36. Lutwak, E., Yang, D., Zhang, G.: On the $L_{p}$-Minkowski problem. Trans. Am. Math. Soc. 356(11), 4359-4370 (2004)

37. Lutwak, E., Yang, D., Zhang, G.: Volume inequalities for subspaces of $L_{p}$. J. Differ. Geom. 68, 159184 (2004)

38. Lutwak, E., Yang, D., Zhang, G.: $L_{p}$ John ellipsoids. Proc. Lond. Math. Soc. 90, 497-520 (2005)

39. Lutwak, E., Yang, D., Zhang, G.: Optimal Sobolev norms and the $L_{p}$ Minkowski problem. Int. Math. Res. Not., 1-21 (2006) 
40. Lutwak, E., Yang, D., Zhang, G.: Volume inequalities for isotropic measures. Am. J. Math. 129, 1711-1723 (2007)

41. Lutwak, E., Yang, D., Zhang, G.: Orlicz projection bodies. Adv. Math. 223, 220-242 (2010)

42. Lutwak, E., Yang, D., Zhang, G.: Orlicz centroid bodies. J. Differ. Geom. 84, 365-387 (2010)

43. Meyer, M., Werner, E.: On the p-affine surface area. Adv. Math. 152, 288-313 (2000)

44. Minkowski, H.: Allgemeine Lehrsätze über die konvexen Polyeder. Nachr. Ges. Wiss. Göttingen 198219 (1897)

45. Minkowski, H.: Volumen und Oberfläche. Math. Ann. 57, 447-495 (1903)

46. Paouris, G.: On the $\psi_{2}$-behaviour of linear functionals on isotropic convex bodies. Stud. Math. 168, 285-299 (2005)

47. Paouris, G.: Concentration of mass on convex bodies. Geom. Funct. Anal. 16, 1021-1049 (2006)

48. Paouris, G.: Concentration of mass on isotropic convex bodies. C. R. Math. Acad. Sci. Paris 342, 179-182 (2006)

49. Schneider, R.: Convex Bodies: The Brunn-Minkowski Theory. Cambridge University Press, Cambridge (1993)

50. Schütt, C., Werner, E.: Surface bodies and p-affine surface area. Adv. Math. 187, 98-145 (2004)

51. Stancu, A.: The discrete planar $L_{0}$-Minkowski problem. Adv. Math. 167, 160-174 (2002)

52. Stancu, A.: On the number of solutions to the discrete two-dimensional $L_{0}$-Minkowski problem. Adv. Math. 180, 290-323 (2003)

53. Stancu, A.: The necessary condition for the discrete $L_{0}$-Minkowski problem in $\mathbb{R}^{2}$. J. Geom. 88, 162-168 (2008)

54. Umanskiy, V.: On solvability of two-dimensional $L_{p}$-Minkowski problem. Adv. Math. 180, 176-186 (2003)

55. Werner, E., Ye, D.: New $L_{p}$ affine isoperimetric inequalities. Adv. Math. 218, 762-780 (2008)

56. Werner, E., Ye, D.: Inequalities for mixed $p$-affine surface area. Math. Ann. 347(3), $703-737$ (2010) 